\title{
Research on Unscrambling Sports Testing Data and Enhancing Physical Quality of Students
}

\author{
Lei $\mathrm{Li}^{1, \mathrm{a}}$ \\ ${ }^{1}$ Yunnan College of Business and Management, Kunming, 650000, China \\ ${ }^{\mathrm{a} e m a i l}$
}

Keywords: Sports testing data, Physical quality, Physical education

\begin{abstract}
The physical quality of the students is directly related to the health of the people of the country and is of great significance. The indexes of students' physical quality, such as flexibility, speed and endurance, can be obtained through sports tests. We carefully analyze the sports testing data of the students, and gives countermeasures to enhance students' physical quality to provide some references for the relative researchers.
\end{abstract}

\section{Introduction}

In the social environment of the students to read a galaxy of talents, the competition is extremely fierce, exam. Physical exercise time is reduced, the amount of exercise is seriously insufficient. This has directly led to the decline of students' physical fitness. In view of the previous domestic research results, we can try to introduce the physical motor function training into the students' physical education. Therefore, this paper takes the students as the object, carries on the experiment research on the physical function training of the body, so as to promote the students' various physical qualities. According to the students' physical structure, muscle and bone characteristics, this paper sorted out the exercises for students' physical function training. Physical fitness is the ability of a person to perform certain movements in sports. Such as speed, quality is the ability to express rapid movement. Physical fitness generally includes strength, speed, stamina, flexibility and agility. The traditional physical training including general physical training and physical training, general physical training is often said that the basic physical training, physical exercise is the use of various methods and means to improve the system performance, the load of each organ of the human body, the development of the athletes' physical quality, improve the athlete's body shape training mode. Special physical fitness training refers to various exercises combined with sports. It can provide some more targeted training methods for school physical education and after-school training. At the same time, it can also promote the application and promotion of physical function training in the physical education at the grass-roots level. Physical fitness refers to the quality of the human body. It is a comprehensive and relatively stable feature of the human body, which is based on heredity, including physiological structure, psychological factors and morphological structure.

\section{Interpretations of Sports Testing Data of Students}

Data Analysis of Flexibility Quality. In physical training, it is possible to make training more scientific only when it is in accordance with the physiological and anatomical features of the human body. Flexible quality refers to the ability of each joint of the human body to move in different directions, as well as the stretching ability of soft tissues such as muscles and ligaments. Soft refers to the muscles and ligaments elongated range, toughness refers to the strength of muscles and ligaments, control joints without injury maximum range of motion. The soft and tough combination is flexible, and its ability to play is called a flexible quality. It shows the rotational range of motion through the joints around a certain axis of motion. Before and after the experiment, the sitting position of the experimental group was $8.26 \pm 5.99 \mathrm{~cm}$ and $13.24 \pm 6.11 \mathrm{~cm}$, and the two groups of data before and after the experiment were tested by $\mathrm{T}$, and the test results were significantly improved. In the control group, the test results were $6.06 \pm 4.21 \mathrm{~cm}$ and $12.68 \pm 5.90 \mathrm{~cm}$ before and after sitting position 
flexion test. After the T test, the test scores also improved significantly. After test, the results of the experimental group and the control group were $13.24 \pm 6.11 \mathrm{~cm}$ and $12.68 \pm 5.90 \mathrm{~cm}$. After the T test, the test scores were not significantly different. Flexibility is the ability to express the joints, ligaments, and muscles in all directions. Flexibility exercises are varied, static stretching, dynamic stretching, PNF, etc., all belong to the movement of the integration and pull. The general physical education adopts the static stretching, and also has the intersection with the bodily function training practice method, therefore the experimental effect is not remarkable. But it is worth the dynamic tensile body function training of action preparation is not only stretching the muscles and the development of joint flexibility, is more important in the stretching process also increased muscle flexibility and explosive force, the motion organs can more directly into the working state, is of great help to improve the ability of muscle work.

Data Analysis of Speed Quality. Speed quality refers to the ability of the human body to move rapidly. Rapid motion reflects the acceleration and maximum speed of the body's movements. Reaction speed refers to the ability of the human body to respond quickly to various signals. Since athletes respond differently to different types of signals, athletes often respond to specific signals according to the characteristics of different events. Such as sprint, swimming and other periodic racing events, athletes mainly receive auditory signals, while table tennis players mainly through the reception of visual signals to make technical and tactical responses. In the male and female statistics before and after the experiment and after the test, the experimental group and the control group between the data, the boys' grades have improved, and the experimental group and the control group after the test results have no significant difference. Girls are different, the results of the control group show that before and after the experiment has not significantly improved, and compared with the experimental group, there is a significant difference. In the contrast between the actual teaching observation and the experimental data, it is found that the performance of the male dash is improved, whether it is the displacement, the speed or the movement speed, and the practice and improvement of the force and explosive force cannot be separated. The control group did not improve the achievement of girl's big problem is that in the process of practice strength and explosive force, girls fatigue, the traditional strength training, increasing focus on single muscle or muscle strength of the model is inconsistent. While the experimental group of girls scores increased significantly because of the body movement function in the training process, strengthened the economic running action, to the development of muscle strength through exercise, the size of the muscles joint coordination movement, so the performance is more obvious.

Data Analysis of Endurance Quality. Endurance quality refers to the ability of a body to maintain a particular intensity, load, or quality of action for a specified period of time. A certain time refers to the regulation of specific sports time. Maintaining a particular intensity or quality of movement is the embodiment of endurance levels. Endurance levels increase as a result of longer periods of time to maintain a particular intensity or action quality, or to withstand higher intensity over a given period of time. Athletes must maintain good athletic strength or exercise quality throughout the race, and must have good endurance qualities. The students in the experimental group and the control group improved significantly after training. The average scores of the experimental group before and after the experiment were improved by 0.65 seconds, and the control group improved by 0.15 seconds before and after the experiment. The experimental group and the control group after the experiment, the results were $8.13 \pm 0.84$ seconds and $8.67 \pm 0.17$ seconds, after the $\mathrm{T}$ test, there was significant difference $(\mathrm{P}<.05)$. It shows that the training method of the experimental group is more helpful to improve the students' speed quality. In the male and female statistics before and after the experiment and after the test, the experimental group and the control group between the data, the boys' grades have improved, and the experimental group and the control group after the test results have no significant difference. Girls are different, the results of the control group show that before and after the experiment has not significantly improved, and compared with the experimental group, there is a significant difference. In the contrast between the actual teaching observation and the experimental data, it is found that the performance of the male 50-meter dash is improved, 
whether it is the displacement, the speed or the movement speed, and the practice and improvement of the force and explosive force cannot be separated. The control group did not improve the achievement of girl's big problem is that in the process of practice strength and explosive force, girls fatigue, the traditional strength training, increasing focus on single muscle or muscle strength of the model is inconsistent.

\section{Countermeasures of Enhancing Physical Quality of Students}

Strengthen Ideological Education. Consciousness decides behavior. We must strengthen the students to participate in physical exercise, develop good exercise habits of ideological education, and use various ways to pass on information to students' physical exercise, and form a good atmosphere for physical exercise. Because of the energetic, strong thirst for knowledge, the use of theoretical knowledge and improve students' understanding ability, to improve students explain the role and significance of physical fitness through exercise, guide the students interested in physical exercise to the benign development, make students understand what to learn, what role can help them get what harvest. Such as: physical exercise can enhance physical fitness, promote the body's normal development, but also can make the body symmetry, the practice results and students pursue bodybuilding together, so that students can consciously long-term exercise, and gradually develop a lifelong habit of exercise. The human body is the same, regular exercise will enhance physical strength, improve the body's resistance to disease, promote the organ systems of the body to enhance physical fitness, The new supersedes the old., improve the health level, so it is very important to the maintenance of the movement process of life, teenagers are in the growth and development period, need more exercise, the kind of "three days fishing, two days of the net" approach is not going to have any success, whether it is learning and mastering the basic skills, or the development of every kind of physical quality, the need to exercise regularly, in order to obtain the desired effect. We know that quality training is tedious, and it's really boring for students to run and jump for a long time. No one will keep it long. To maintain the students' enthusiasm for exercise, we should use various scientific methods to stimulate the enthusiasm of students. Such as sprint, can be in the form of group games, such as a variety of relay running, listening to signal chase, running a variety of starting positions, such as fast running.

Reform Physical Education Courses. The main factors that restrict the healthy development of the physical education curriculum are the lack of public sports courses, the lack of teachers and the lack of venues and facilities. We should encourage young teachers' professional transformation to offer more students interested in the project, such as yoga, roller skating, sports and other new courses, attracting students take the initiative to actively choose their favorite sports items, so as to form a good habit of physical exercise. Media intervention has promoted the development of physical education in Higher Vocational colleges. Through radio, newspapers, Internet, etc., find the matching group, the information related to sports, especially the sport has the value of using the right direction media, to create a good atmosphere. Specific practices, such as posters can be posted on major university websites set up sports columns, newspapers published the corresponding information. Conditions can also make some games and performances broadcast through television, so that people can fully understand the sport, and fell in love with the sport in the understanding, the actual participation, so as to achieve the purpose of the promotion of sports. We can carry out targeted sports program selection and practice, at the same time to exercise control and control of time, master the rules, group practice and gradual progress. Step like according to test data, we can determine the amount of exercise can also determine the movement time and movement project, intense antagonism in basketball as the representative, we can step detection data of high students in the first place, by students after progressive training. For the vital capacity of the data, we can also high capacity students in long-distance running in the advantages of the students after the effective exercise and promotion. According to the data of different teachers, to arouse students' enthusiasm, so as to obtain the final harvest, is their own benefit, for the practical operation of the requirements and specific action display, and to meet the standards, to achieve the target of learning, it is not only the feedback 
of learning theory, it is the students' practical operation demonstration and a test of willpower exercise.

Perfect Evaluation Mechanism. First of all, to establish a scientific evaluation of the guiding ideology, to research on the connotation of physical quality education, physical quality in seize the latent, basic characteristics, development, status, school sports in the role of quality education, specific training objectives of school physical education, make the evaluation objectives consistent with the goal of education, and to the design of physical education evaluation based on index system. Secondly, the continuous expansion of evaluation content: the content of PE teaching evaluation should pay attention to multiple evaluation, including cognitive, technical, technical and emotional content of three aspects, rather than a single technical skills assessment or health test. Thirdly, a variety of evaluation methods are used in a comprehensive way. In the past physical education evaluation activities, we pay more attention to summative evaluation, because this method is carried out at the end of unit or phase learning. Therefore, losing the effective feedback function of evaluation can not only stimulate students' learning, improve their learning effect, but also help teachers to improve their teaching. Based on the above, we should change the method of summative evaluation, but adopt the combination of diagnostic, formative and summative evaluation. Qualitative evaluation and quantitative evaluation are combined. In physical education, there are a lot of human factors, like the attitude of the students, moral character, psychological quality, exercise ability index with qualitative characteristics, it is difficult to quantify, such as the neglect of education evaluation is difficult to quantify these indicators is not comprehensive, lack of scientific. Therefore, quantitative evaluation and qualitative evaluation must be used together. Scientific evaluation focuses on motivation. In the evaluation, individual evaluation should be carried out, the content and method of evaluation should be changed, and the results of evaluation should be fully used to motivate students so that each student can see his progress and improvement.

\section{Conclusion}

Therefore, a careful interpretation of the test data of physical quality can help us to develop a reasonable and effective way to improve the students' physique through the analysis of data. The comparison of the data provides a basis for each student to understand the physical condition of the body. It also points out the direction for how to improve the overall quality of students.

\section{References}

[1] Chen Haiqiong, Wu Yuhua, Comparative Study about Physical Fitness of University Students in Gannan Normal University - Base on the Data of Physical Fitness Test in 2013 [J]. Journal of Gannan Normal University, 2014(3): 91-95.

[2] Wang Wenhong, Sun Yiran, Du Peng, Data Analysis of Students' Physical Health Testing in Vocational Colleges in Yunnan Province: Testing Performance of Kunming Metallurgy College in 2014 as an Example [J]. Journal of Kunming Metallurgy College, 2015, 31(2): 47-51.

[3] Wang Hui, Zhang Yuhong, The Research on the Factors and Countermeasures of College Students “Three Big” Physical Fitness Test Data Accuracy [J]. Journal of Jilin Institute of Physical Education, 2012, 28(4): 69-72.

[4] Xue Hongqiang, On the Application of Data Mining in Students’ Physical Fitness Test [J]. Journal of Xi'an Aeronautical University, 2014, 32(6): 87-90. 